\title{
A Heuristic for a Mixed Integer Program using the Characteristic Equation Approach
}

\author{
Philimon Nyamugure \\ Department of Statistics and Operations Research \\ National University of Science and Technology \\ Box AC 939, Ascot, Bulawayo, Zimbabwe \\ E-mail: philimon.nyamugure@ nust.ac.zw \\ Elias Munapo \\ School of Economics and Decision Sciences \\ North West University, Mafikeng Campus \\ Mafikeng, South Africa \\ E-mail: emunao@gmail.com \\ 'Maseka Lesaoana \\ Department of Statistics and Operations Research \\ University of Limpopo, Private Bag X1106, Sovenga 0727, South Africa \\ E-mail: Lesaoana.Maseka@ul.ac.za \\ Santosh Kumar* \\ Department of Mathematics and Statistics \\ University of Melbourne, Melbourne, Australia \\ E-mail: santosh.kumarau@gmail.com \\ *Correspondence author
}

(Received October 21, 2016; Accepted November 9, 2016)

\begin{abstract}
While most linear programming (LP) problems can be solved in polynomial time, pure and mixed integer problems are NP-hard and there are no known polynomial time algorithms to solve these problems. A characteristic equation (CE) was developed to solve a pure integer program (PIP). This paper presents a heuristic that generates a feasible solution along with the bounds for the NP-hard mixed integer program (MIP) model by solving the LP relaxation and the PIP, using the CE.
\end{abstract}

Key words - Mixed integer program, Pure integer program, Characteristic equation, LP relaxation.

\section{Introduction}

An integer linear program is a linear program which is further constrained by integer restrictions on some or all variables. When all variables are integer restricted, it is called a pure integer program (PIP) model and when only some of the variables are restricted to integer values, it becomes a mixed integer program (MIP) model. Integer programming (IP) models frequently arise in human resource planning, facility location, assignment problems, production planning, time-tabling, warehouse location, scheduling and capital budgeting, just to mention a few. While most linear programming (LP) problems can be solved in polynomial time, PIP and MIP are NP-complete problems, which have no known polynomial time algorithm to solve them. In this paper, the PIP is solved by using the characteristic equation (CE) with the hope that this approach may provide insight into MIP solution procedures and applications. Generally, MIP problems have been solved 
International Journal of Mathematical, Engineering and Management Sciences

Vol. 2, No. 1, 1-16, 2017

https://dx.doi.org/10.33889/IJMEMS.2017.2.1-001

using the LP-based branch and bound (BB) solvers or with stochastic search-based solvers (Noraini and Geraghty, 2011). In reality MIP solvers have implemented more sophisticated versions denoted by branch and cut (BC) algorithms (Sen and Sherali, 2006). With the increase in the application of both PIP and MIP, it is of paramount importance that methods sought are capable of finding a global optimal solution. The major disadvantages of existing methods like round off errors, and creation or emergence of many sub-problems (branches), is the time taken to obtain the optimal solution and failure to obtain global optimal solutions. These justify the need to find better approaches for MIP problems.

In this paper, a hybrid of the existing approaches for solving the LP and PIP models has been used for solving the MIP model. A PIP has been solved by using a descending hyper-plane that was developed by Kumar et al. (2007), which was later renamed a characteristic equation (CE) by Kumar and Munapo (2012). For the MIP model, the LP solution acts as an upper bound (UB) and the PIP solution as the lower bound (LB). In a MIP model, one has to deal with integer restricted as well as continuous variables, requiring distribution of the available resources for these two types of variables. This aspect of distribution has been addressed by the CE, since ordered optimal solutions can be obtained by the CE for a PIP model. The proposed method generates a good feasible solution with bounds and eliminates rounding off errors and dealing with sub-problems as is commonly required in existing BB methods.

The paper has been organized in six sections. Briefly the literature has been reviewed in Section 2 and solution approaches have been summarized in Section 3. Development of the proposed method is presented in Section 4. Numerical illustrations have been presented in Section 5. Finally, the paper has been concluded in Section 6.

\section{Literature Review}

Mixed integer programming is a widely used formulation for dealing with hard optimization problems. Many practical optimization problems arising from real-world applications (e.g., scheduling, project planning, transportation, telecommunications, economics and finance, timetabling, etc.) have been formulated as MIPs (Vielma, 2015). A wide range of other problems can also be modelled as mixed integer linear programs. In many cases the resulting MIP can either be too weak or too large to be solved effectively by the state-of-the-art solvers (Vielma, 2015). Mathematical programming has been used in the literature to tackle some problems as a LP model (Yildiz and Vielma, 2013); some as a PIP model (Laesanklang et al., 2015); and some as a MIP model (Borsani et al., 2006; Bredstrom and Rönnqvist, 2007; Dohn et al., 2009; Rasmussen et al., 2012). Mixed integer conic programming (MICP) is a generalization of mixed integer linear programming. A sub-additive dual for MICP problems was constructed under a simple condition on the primal problem (Morán et al., 2012). An encoding formulation framework that encompasses and expands existing approaches to mitigate this behaviour generalizes the incremental formulation for piecewise linear functions to any finite union of polyhedral with identical recession cones (Burer and Letchford, 2012).

\section{Solution Approaches}

\subsection{The Simplex Method}

Essentially, the simplex algorithm solves a LP problem by searching along the edges of the convex polyhedron or the space of feasible solutions. From the LP theory, it is known that the optimal solution is either an extreme point or an extreme ray of the convex polytope. As such, it is enough to only search the boundary of the LP's feasible region. Moreover, the MIP is a method that has its 
International Journal of Mathematical, Engineering and Management Sciences

Vol. 2, No. 1, 1-16, 2017

https://dx.doi.org/10.33889/IJMEMS.2017.2.1-001

roots in LP, where MIP is simply an extension of the LP problem that requires integer restricted variables (Fiorin et al., 2012).

\subsection{Branch and Bound}

The approach for tackling a MIP is the branch and bound (BB) algorithm, which relies on the iterative solution of the LP relaxation. The reason for dropping integer constraints is to be able to use LP general-purpose solution methods (Kaibel, 2011). The standard way to represent a choice between $n$ alternatives in a mixed integer programming model is through $n$ binary variables that add up to one. Unfortunately, this approach commonly leads to unbalanced BB trees and diminished solver performance (Dey and Vielma, 2010).

\subsection{Branch and Cut}

The branch and cut (BC) algorithm incorporates the cutting-plane algorithm in the BB scheme, and the idea behind integrating the two algorithms is that LP relaxations do not naturally well approximate the convex hull of mixed integer solutions to MIPs (Jozefowiez et al., 2012). Research in this area is continuing, along with the development of sophisticated new software packages that incorporate these new techniques.

\subsection{Decomposition Techniques}

Decomposition techniques are another good alternative to apply exact optimization methods to solve large integer programming formulations. The basic idea here is to transform or split the problem into smaller sub-problems. This technique has been applied in various problem domains. For example, Benders' (1962) decomposition was used to produce solutions for large instances of the aircraft routing and crew scheduling problems (Laesanklang et al., 2015). Decomposition techniques have also been applied within heuristic approaches using some form of clustering. For example, Jozefowiez et al. (2012) tackled a large vehicle routing problem by decomposing it into sub-problems. The sub-problem size is controlled by splitting a large sub-problem to shrink the corresponding cluster.

\subsection{Stochastic Programming}

Stochastic programming becomes an increasingly popular technique to model decision making under uncertainty. It is able to model uncertainties in a flexible way and impose real-world constraints relatively easily (Sherali and Adams, 1990). Sourd and Spanjaard (2008) proposed a novel decomposition method based on the particular structure of the problem concerned. It decomposes the problem geographically into security and bond sub-problems, which are then further broken into smaller sub-problems.

\subsection{Lagrangian Relaxation}

Lagrangian (sometimes spelled as Lagrangean) relaxation is a popular relaxation technique to compute bounds on the optimal solution of a MIP (Sridhar, 2014). These algorithms are either combinatorial or LP-based. The combinatorial algorithms include greedy algorithms, randomized algorithms and reduction to problems with known poly-time algorithms (Sridhar, 2014).

\section{Proposed Method}

\subsection{Mathematical Development for The Proposed Method}

Consider a general mixed integer programming problem 
International Journal of Mathematical, Engineering and Management Sciences

Vol. 2, No. 1, 1-16, 2017

https://dx.doi.org/10.33889/IJMEMS.2017.2.1-001

$$
\left.\begin{array}{l}
\text { Min/Max } C^{T} X \\
\text { subject to : } \\
A X \geq /=I \leq b \\
X \geq 0 \\
X_{1} \in Z
\end{array}\right\}
$$

where $C^{T}$ is $(1 \mathrm{x} n), X$ is $(n \times 1), A$ is $(m \times n)$ and $b$ is $(m \times 1)$. Let $n_{1}<n$ represent integer restricted non-negative variables and the remaining $\left(n-n_{1}\right)$ variables are such that $x_{j} \geq 0$. In equation (1), $X_{1}$ represents integer restricted non-negative variables in $X$, implying that $X_{1} \geq 0$. The MIP relaxation, i.e. LP of the model (1) is given by

$$
\left.\begin{array}{l}
\operatorname{Min} / \operatorname{Max} C^{T} X \\
\text { subject to : } \\
A X \geq I=/ \leq b \\
X \geq 0
\end{array}\right\}
$$

The pure integer programming model of (1) is given by

$$
\left.\begin{array}{l}
\text { Min/Max } C^{T} X \\
\text { subject to }: \\
A X \geq /=/ \leq b \\
X \geq 0 \text { and integer }
\end{array}\right\}
$$

After obtaining the PIP solution to (3), one can also develop a modified LP from the given MIP (equation (1)) when all integer restricted variables are replaced by their values and the problem reduces to $\left(n-n_{1}\right)$ variables, where all these variables are non-negative restricted real variables. Here onwards, we will call it a modified LP model.

The three problems (1), (2) and (3), and the modified LP have close relationships among themselves, which can be used to develop a method for solving the MIP model (1). Some of these relationships that may be of immediate interest to us are discussed next.

\section{Observation 1}

The LP relaxation model (2) is a least constrained model among models (1), (2) and (3), hence the LP optimal solution of model (2) will be an upper bound to the MIP model (1).

\section{Observation 2}

A feasible solution to the PIP model (3) will also be feasible to the MIP model (1).

Proof - Since a feasible solution X to model (3) will also satisfy the requirement for the MIP model (1), where $n_{1}$ of the $n$ variables are required to have integer restricted values, it then follows that 
International Journal of Mathematical, Engineering and Management Sciences

Vol. 2, No. 1, 1-16, 2017

https://dx.doi.org/10.33889/IJMEMS.2017.2.1-001

all feasible solutions to the PIP model (3) will also be feasible to the MIP model (1). Therefore, the PIP optimal solution will act as a lower bound to the MIP model (1).

\section{Observation 3}

The MIP model involves two-way distribution of resources. the first distribution of resources involves the division of the resource vector $b$ among the integer and continuous variables and the second distribution gives rise to values of the basic variables. the distribution of the resource vector $b$ is achieved by determining ordered optimal solutions to the PIP model. These ordered optimal solutions can be obtained by using the characteristic equation developed by Kumar et al. (2007), and Kumar and Munapo (2012). The distribution of resources to continuous variables is obtained by the LP.

\section{Observation 4}

The optimal PIP solution $X_{\text {PIPopt }}$ has a property that if $x_{j}=\beta_{j}$ is an element of this optimal integer solution, then $x_{j}=\beta_{j}+1$ along with other variables at their optimal values will always lead to an infeasible solution.

\subsection{The Method}

In the proposed approach, for the given MIP, we first develop two parallel LP and PIP models. The optimal value of the relaxed objective is denoted by $Z_{L P}$ which acts as the upper bound to the given MIP. For any optimization problem $Z$, let $F(Z)$ denote its set of feasible solutions. The only requirement for $Z_{L P}$ to be a valid relaxation of $Z$ is $F(Z) \subseteq F\left(Z_{L P}\right)$ which is true in this case. The relaxed problem is easier to solve than the original problem, and the $Z_{L P}$ gives the upper bound (Geoffrion and Marsten, 1972). From the optimal solution of $Z_{L P}$ a characteristic equation (CE) is formed to resolve the PIP model (3), where all variables are restricted to integer values. The advantage of the CE is that it can provide the best, second best, third best solutions, etc. for the PIP model (3). The optimal PIP solution acts as the LB to the given MIP model. The gap between the UB given by $Z_{L P}$ and the LB given by $Z_{P I P}$ can be decreased by the LP modified model that can be formulated by substituting values of the integer restricted variables in the MIP model (1) and solving the remaining model as a LP model in $\left(n-n_{1}\right)$ variables. The combined solution will be a feasible solution to the MIP model (1). This will give rise to an improved LB, denoted by $Z_{M I P}$. If the difference $\left(Z_{L P}-Z_{M I P}\right)$ is insignificant or is zero then either the optimal solution or near optimal solution to the MIP is obtained.

\subsection{The Characteristic Equation}

The characteristic equation (CE) is obtained from the final table of the LP relaxation. It is a mapping of the integer hyper-plane on feasible integer points and the mapping of the objective function on interior integer points in a descending order.

The CE is based on the following three basic ideas

(i) The objective value must be an integer.

(ii) Non-basic variables are either zero (as in the LP solution) or if some of them are not zero, they must be an integer quantity.

(iii) Basic variables are also functions of the non-basic variables, and for the non-zero nonbasic variables, the basic variables must also become integer values in a PIP model. 
International Journal of Mathematical, Engineering and Management Sciences

Vol. 2, No. 1, 1-16, 2017

https://dx.doi.org/10.33889/IJMEMS.2017.2.1-001

The major advantage of using the CE is that convergence is guaranteed and it can be used to obtain ordered optimal solutions for the PIP problem. However, the solution of the CE can be a challenge, and also if there are more than one solution to the CE, all those solutions have to be tested for integer solutions. The LP extreme points are an intersection of at most $m$ constraints and the LP optimal solution is an intersecting point of one more hyper-plane represented by the objective function. The $\mathrm{CE}$ is a mapping of the hyper-plane on feasible integer points. From the optimal relaxation LP solution, one can write the objective function row as

$$
\left(\frac{D}{D}\right) Z+\frac{\beta_{1} s_{2}+\beta_{2} s_{2}+\ldots \ldots \ldots+\beta_{k} s_{k}}{D}=\frac{(R+i D)}{D}
$$

where $k$ represents the number of non-basic variables, $\beta_{j}$ represents the integer coefficients, $D$ is the lowest common factor for all terms and $R$ is the remainder in the RHS value. The CE is then given by

$\beta_{1} s_{1}+\beta_{2} s_{2}+\ldots \ldots \ldots \ldots \ldots \ldots+\beta_{k} s_{k}=R+i, \quad i=0,1,2,3 \ldots \ldots \ldots$

When the LHS is equal to the RHS, the objective function $\mathrm{Z}$ is guaranteed to be an integer value. Since we are looking for an integer solution to the CE, we also got the condition (2) in Section 4.3 satisfied. For the solution to be an acceptable feasible solution, the solution of the characteristic solution must also convert the values of the basic variables to non-negative integer values. The next best solution is obtained by further reduction in the value of the objective function, which is possible by increasing the value of $i$.

It may also be noted that when the PIP model (3) is solved for the optimal solution, all variables have non-negative integer values. We substitute from this solution, values of $n_{1}$ integer restricted values in the MIP model (1) and get a modified LP in $\left(n-n_{1}\right)$ real variables, which is a LP model and can be solved by any known method. The combined integer restricted values from the PIP and LP solution for the modified LP gives a feasible solution to the MIP, hence acts as a LB.

\subsection{Algorithmic Steps of the Method}

The method is comprised of the following steps.

Step 1. Solve the relaxed LP model (2), find the value of $Z_{L P}$ which will be an UB to the given problem (1).

Step 2. Obtain the characteristic equation from the solution of $Z_{L P}$. Set $k=1$.

Step 3. Solve the characteristic equation and obtain $k$ th best integer solution for minimum $i$. This will be a solution to the PIP, i.e. when all are integer restricted variables.

Step 4. The PIP solution from Step 3 will be a lower bound to the given MIP. Let this LB be denoted by $Z_{P I P}$.

Step 5. If $\left(Z_{L P}-Z_{P I P}\right)$ equal to zero, or approximately equal to zero go to Step 10. Else go to Step 6.

Step 6. Substitute the integer solution for the integer restricted variables obtained from Step 3 into the original MIP model (1) and get a modified LP in $\left(n-n_{1}\right)$ variables.

Step 7. Solve the LP in $\left(n-n_{1}\right)$ real variables obtained at Step 6. 
International Journal of Mathematical, Engineering and Management Sciences

Vol. 2, No. 1, 1-16, 2017

https://dx.doi.org/10.33889/IJMEMS.2017.2.1-001

Step 8. Combine the integer solution of Step 3 for the integer variables and real solution for the real variables from Step 7 to get a feasible solution to the MIP. This value is likely to be less than the UB and more than the LB and if the difference is insignificant, one can stop the search, else one has to improve the feasible solution. Check if this solution can be declared as the optimal solution. If yes, go to Step 10, else go to Step 9.

Step 9. Set $k=k+1$ and go to Step 3.

Step 10. Conclude the search process as the optimality condition has been satisfied.

\section{Numerical Illustrations}

\subsection{Example 1}

This example is taken from Hillier and Lieberman (2001).

$\operatorname{Max} 4 x_{1}-2 x_{2}+7 x_{3}-x_{4}$

subject to :

$x_{1}+5 x_{3} \leq 10$

$x_{1}+x_{2}-x_{3} \leq 1$

$6 x_{1}-5 x_{2} \leq 0$

$-x_{1}+2 x_{3}-2 x_{4} \leq 3$

$x_{1}, x_{2}, x_{3} \geq 0$ and $\in Z$

$x_{4} \geq 0$

Using LIPS programme, the LP solution found is shown in Table 1 and the final simplex solution is shown in Table 2.

\begin{tabular}{|l|c|c|c|}
\hline Variable & Value & Obj. Cost & Reduced Cost \\
\hline$x_{1}$ & 1.25 & 4 & 0 \\
\hline$x_{2}$ & 1.50 & -2 & 0 \\
\hline$x_{3}$ & 1.75 & 7 & 0 \\
\hline$x_{4}$ & 0 & -1 & 1 \\
\hline
\end{tabular}

Table 1. Solution to the LP problem

$\mathrm{Z}=14.25$.

\begin{tabular}{|c|c|c|c|c|c|c|c|c|c|}
\hline Basis & $x_{1}$ & $x_{2}$ & $x_{3}$ & $x_{4}$ & $s_{5}$ & $s_{6}$ & $s_{7}$ & $S_{8}$ & RHS \\
\hline$x_{2}$ & 0 & 1 & 0 & 0 & 0.1 & 0.5 & -0.1 & 0 & 1.50 \\
\hline $\mathrm{S} 8$ & 0 & 0 & 0 & -2 & $-17 / 60$ & $7 / 12$ & $7 / 60$ & 1 & 0.75 \\
\hline$x_{1}$ & 1 & 0 & 0 & 0 & $1 / 12$ & $5 / 12$ & $1 / 12$ & 0 & 1.25 \\
\hline$x_{3}$ & 0 & 0 & 1 & 0 & $11 / 60$ & $-1 / 12$ & $-1 / 60$ & 0 & 1.75 \\
\hline Obj. & 0 & 0 & 0 & -1 & $-17 / 12$ & $-1 / 12$ & $-5 / 12$ & 0 & 14.25 \\
\hline
\end{tabular}

Table 2. Final table of the simplex iterations 
International Journal of Mathematical, Engineering and Management Sciences

Vol. 2, No. 1, 1-16, 2017

https://dx.doi.org/10.33889/IJMEMS.2017.2.1-001

From the LP solution, the UB $=14.25$ for the given MIP model (6). Now we solve the model (6) again as a PIP and for that the CE will be required, which is given by (7)

$$
12 x_{4}+17 s_{5}+s_{6}+5 s_{7}=3+12 i \quad i=0,1,2, \ldots \ldots \ldots, 14
$$

The non-basic variables $x_{4}, s_{5}, s_{6}$ and $s_{7}$ have to move from the current value zero to some nonnegative integer value such that (7) remains satisfied for the minimum $i$ and also converts the current basic variables from the real values to integer values. From Table 2, the relations between the basic and non-basic variables are given by

$$
\left.\begin{array}{l}
x_{1}=1.25-\left(\frac{1}{12}\right) s_{5}-\left(\frac{5}{12}\right) s_{6}-\left(\frac{1}{12}\right) s_{7} \\
x_{2}=1.5-0.1 s_{5}-0.5 s_{6}+0.1 s_{7} \\
x_{3}=1.75-\left(\frac{11}{60}\right) s_{5}+\left(\frac{1}{12}\right) s_{6}+\left(\frac{1}{60}\right) s_{7} \\
s_{8}=0.75+2 x_{4}+\left(\frac{17}{60}\right) s_{5}-\left(\frac{7}{12}\right) s_{6}-\left(\frac{7}{60}\right) s_{7}
\end{array}\right\}
$$

For $i=0$, equation (7) becomes

$$
12 x_{4}+17 s_{5}+s_{6}+5 s_{7}=3
$$

The only integer solution from equation (9) is $x_{4}=0, s_{5}=0, s_{6}=3$ and $s_{7}=0$. Putting this solution in (8), we have $x_{1}=x_{2}=x_{4}=0, x_{3}=2, s_{8}=-1$, which is not feasible, hence one has to try the next value $i=1$, for which the $\mathrm{CE}$ is

$$
12 x_{4}+17 s_{5}+s_{6}+5 s_{7}=15
$$

Equation (10) has five integer solutions, as follows

$$
\begin{array}{ll}
s_{5}=0, s_{6}=15, s_{7}=0, x_{4}=0 & \text { No feasible integer solution } \\
s_{5}=0, s_{6}=3, s_{7}=0, x_{4}=1 & x_{1}=x_{2}=0, x_{3}=2, x_{4}=1 \text { and } Z=13 \\
s_{5}=0, s_{6}=5, s_{7}=2, x_{4}=0 & \text { No feasible integer solution } \\
s_{5}=0, s_{6}=10, s_{7}=1, x_{4}=0 & \text { No feasible integer solution } \\
s_{5}=0, s_{6}=0, s_{7}=3, x_{4}=0 & \text { No feasible integer solution }
\end{array}
$$

From the above the best integer solution gives a $\mathrm{Z}$ of 13 and this value forms a $\mathrm{LB}$, and the UB is 14.25. Substituting the integer values for the integer restricted variables from the above solution $\left(x_{1}=x_{2}=0, x_{3}=2\right)$ into MIP model (6) we obtain, a modified LP in $\mathrm{x}_{4}$. 
International Journal of Mathematical, Engineering and Management Sciences

Vol. 2, No. 1, 1-16, 2017

https://dx.doi.org/10.33889/IJMEMS.2017.2.1-001

$\left.\begin{array}{l}\operatorname{Max} 14-x_{4} \\ \text { subject to : } \\ 4-2 x_{4} \leq 3 \\ x_{4} \geq 0\end{array}\right\}$

Solving (11) trivially gives $x_{4}=\frac{1}{2}$.

Combining the integer solution and the above continuous solution we have an improved solution to the given MIP model, which is given by

$$
x_{1}=x_{2}=0, x_{3}=2, x_{4}=0.5 \text { and } Z=13.5 .
$$

The above solution can be concluded as an optimal solution to the given MIP, as currently we have $13 \leq Z_{\text {MIP }} \leq 14.25$, and a feasible solution of 13.5. Note that the only variation possible is to increase the values of the variables $x_{1}, x_{2}, x_{3}$, but the solution will exceed the current UB. Similarly, an increase in $x_{4}$ will worsen the current solution. Hence an optimal solution has been obtained and the search is terminated.

The above solution can be concluded as an optimal solution to the given MIP, as currently we have $13 \leq Z_{\mathrm{MIP}} \leq 14.25$, and a feasible solution of 13.5 . Note that the only variation possible is to increase the values of the variables $x_{1}, x_{2}, x_{3}$, but the solution will exceed the current UB. Similarly, an increase in $x_{4}$ will worsen the current solution. Hence, an optimal solution has been obtained and the search is terminated.

\subsection{Example 2}

Consider the following MIP

$\operatorname{Max} 2 x_{1}-x_{2}+5 x_{3}+3 x_{4}+4 x_{5}-2 x_{6}+2 x_{7}+3 x_{8}+2 x_{9}-3 x_{10}+x_{11}+2 x_{12}$

subject to :

$$
\begin{aligned}
& 1 x_{1}+4 x_{2}+0 x_{3}-1 x_{4}+5 x_{5}-2 x_{6}-2 x_{7}+4 x_{8}+0 x_{9}+0 x_{10}+3 x_{11}+1 x_{12} \leq 24 \\
& 2 x_{1}+0 x_{2}+2 x_{3}+0 x_{4}+3 x_{5}+1 x_{6}+3 x_{7}+0 x_{8}+0 x_{9}+1 x_{10}+4 x_{11}+0 x_{12} \leq 32 \\
& 4 x_{1}+0 x_{2}+5 x_{3}+0 x_{4}+0 x_{5}+5 x_{6}+0 x_{7}+4 x_{8}+0 x_{9}+1 x_{10}+0 x_{11}+2 x_{12} \leq 20 \\
& 3 x_{1}+1 x_{2}+0 x_{3}+3 x_{4}+2 x_{5}+1 x_{6}+3 x_{7}-2 x_{8}+4 x_{9}+0 x_{10}+2 x_{11}+0 x_{12} \leq 30 \\
& 0 x_{1}+2 x_{2}+4 x_{3}+0 x_{4}-2 x_{5}+3 x_{6}+0 x_{7}+0 x_{8}+0 x_{9}+1 x_{10}-2 x_{11}+0 x_{12} \leq 22 \\
& 2 x_{1}+0 x_{2}+1 x_{3}+4 x_{4}+1 x_{5}+2 x_{6}+2 x_{7}+0 x_{8}+5 x_{9}+3 x_{10}+1 x_{11}+0 x_{12} \leq 12 \\
& 0 x_{1}+2 x_{2}+0 x_{3}+1 x_{4}+0 x_{5}+2 x_{6}+1 x_{7}+2 x_{8}+0 x_{9}+0 x_{10}+4 x_{11}+5 x_{12} \leq 28 \\
& 1 x_{1}+1 x_{2}+0 x_{3}+0 x_{4}+3 x_{5}+1 x_{6}+0 x_{7}+1 x_{8}+1 x_{9}+2 x_{10}+2 x_{11}+6 x_{12} \leq 36 \\
& 3 x_{1}+0 x_{2}+2 x_{3}+2 x_{4}+1 x_{5}+4 x_{6}-2 x_{7}+0 x_{8}-1 x_{9}+2 x_{10}+0 x_{11}+3 x_{12} \leq 18
\end{aligned}
$$


International Journal of Mathematical, Engineering and Management Sciences

Vol. 2, No. 1, 1-16, 2017

https://dx.doi.org/10.33889/IJMEMS.2017.2.1-001

where ${ }^{x_{1}, x_{3}, x_{6} \geq 0 \text { and integer }}$

and $x_{2}, x_{4} x_{5}, x_{7}, x_{8}, x_{9}, x_{10}, x_{11}, x_{12} \geq 0$

Details of the LP optimal solution are given in the Appendix A, Tables 3 and 4. From the optimal LP solution, the UB for the given MIP is $Z_{L P}=\frac{2528}{57}=44.351$.

The $\mathrm{CE}$ for the $\mathrm{Z}_{\mathrm{PIP}}$ will be given by

$$
\begin{gathered}
245 x_{1}+170 x_{2}+141 x_{4}+429 x_{6}+102 x_{8}+312 x_{9}+468 x_{10}+114 x_{11}+28 S_{13}+40 S_{15} \\
+85 S_{18}+S_{20}=20+57 i
\end{gathered}
$$

The non-basic variables have to move from the current value of zero to some non-negative integer value such that (13) remains satisfied for some value of $i=0,1,2, \ldots \ldots \ldots, 44$. The basic variables can now be obtained as a function of non-basic variables (from the final table given in the appendix) as

$$
\begin{aligned}
& x_{3}=\left(\frac{148}{57}\right)-\left(\frac{47}{57}\right) x_{1}-\left(\frac{4}{57}\right) x_{2}-\left(\frac{2}{19}\right) x_{4}-\left(\frac{17}{19}\right) x_{6}-\left(\frac{16}{19}\right) x_{8}-\left(\frac{2}{19}\right) x_{9}-\left(\frac{3}{19}\right) x_{10} \\
& -\left(\frac{2}{57}\right) S_{13}-\left(\frac{11}{57}\right) S_{15}-\left(\frac{2}{57}\right) S_{18}+\left(\frac{4}{57}\right) S_{20} \\
& x_{5}=\left(\frac{284}{57}\right)-\left(\frac{7}{19}\right) x_{1}-\left(\frac{13}{19}\right) x_{2}-\left(\frac{10}{19}\right) x_{4}+\left(\frac{11}{57}\right) x_{6}-\left(\frac{31}{57}\right) x_{8}-\left(\frac{49}{57}\right) x_{9}-\left(\frac{26}{57}\right) x_{10}-\left(\frac{2}{3}\right) x_{11} \\
& -\left(\frac{10}{57}\right) S_{13}+\left(\frac{2}{57}\right) S_{15}-\left(\frac{10}{57}\right) S_{18}+\left(\frac{1}{57}\right) S_{20} \\
& x_{7}=\left(\frac{42}{19}\right)-\left(\frac{47}{114}\right) x_{1}+\left(\frac{47}{114}\right) x_{2}-\left(\frac{32}{19}\right) x_{4}-\left(\frac{37}{57}\right) x_{6}+\left(\frac{79}{114}\right) x_{8}-\left(\frac{115}{57}\right) x_{9}-\left(\frac{68}{57}\right) x_{10}-\left(\frac{1}{6}\right) x_{11} \\
& +\left(\frac{2}{19}\right) S_{13}+\left(\frac{3}{38}\right) S_{15}-\left(\frac{15}{38}\right) S_{18}-\left(\frac{5}{114}\right) S_{20} \\
& x_{12}=\left(\frac{200}{57}\right)+\left(\frac{1}{57}\right) x_{1}+\left(\frac{10}{57}\right) x_{2}+\left(\frac{5}{19}\right) x_{4}-\left(\frac{5}{19}\right) x_{6}+\left(\frac{2}{19}\right) x_{8}+\left(\frac{5}{19}\right) x_{9}-\left(\frac{2}{19}\right) x_{10} \\
& +\left(\frac{5}{57}\right) S_{13}-\left(\frac{1}{57}\right) S_{15}+\left(\frac{5}{57}\right) S_{18}-\left(\frac{10}{157}\right) S_{20}
\end{aligned}
$$


International Journal of Mathematical, Engineering and Management Sciences

Vol. 2, No. 1, 1-16, 2017

https://dx.doi.org/10.33889/IJMEMS.2017.2.1-001

$$
\begin{aligned}
& S_{14}=\left(\frac{298}{57}\right)+\left(\frac{223}{114}\right) x_{1}+\left(\frac{121}{114}\right) x_{2}+\left(\frac{130}{19}\right) x_{4}+\left(\frac{41}{19}\right) x_{6}+\left(\frac{47}{38}\right) x_{8}+\left(\frac{168}{19}\right) x_{9}+\left(\frac{81}{19}\right) x_{10}-\left(\frac{3}{2}\right) x_{11} \\
& +\left(\frac{16}{57}\right) S_{13}+\left(\frac{5}{114}\right) S_{15}+\left(\frac{203}{114}\right) S_{18}-\left(\frac{7}{114}\right) S_{20} \\
& S_{16}=\left(\frac{164}{57}\right)-\left(\frac{39}{38}\right) x_{1}-\left(\frac{29}{38}\right) x_{2}+\left(\frac{59}{19}\right) x_{4}+\left(\frac{32}{57}\right) x_{6}+\left(\frac{115}{114}\right) x_{8}+\left(\frac{215}{57}\right) x_{9}+\left(\frac{256}{57}\right) x_{10}-\left(\frac{1}{6}\right) x_{11} \\
& +\left(\frac{2}{57}\right) S_{13}-\left(\frac{35}{114}\right) S_{15}+\left(\frac{175}{114}\right) S_{18}+\left(\frac{11}{114}\right) S_{20} \\
& S_{17}=\left(\frac{410}{19}\right)+\left(\frac{142}{57}\right) x_{1}-\left(\frac{176}{57}\right) x_{2}-\left(\frac{12}{19}\right) x_{4}+\left(\frac{55}{57}\right) x_{6}+\left(\frac{130}{57}\right) x_{8}-\left(\frac{74}{57}\right) x_{9}-\left(\frac{73}{57}\right) x_{10}+\left(\frac{2}{3}\right) x_{11} \\
& -\left(\frac{4}{19}\right) S_{13}+\left(\frac{16}{19}\right) S_{15}-\left(\frac{4}{19}\right) S_{18}-\left(\frac{14}{57}\right) S_{20} \\
& S_{19}=\left(\frac{470}{57}\right)+\left(\frac{37}{114}\right) x_{1}-\left(\frac{371}{114}\right) x_{2}-\left(\frac{12}{19}\right) x_{4}-\left(\frac{2}{57}\right) x_{6}-\left(\frac{367}{114}\right) x_{8}+\left(\frac{40}{57}\right) x_{9}+\left(\frac{98}{57}\right) x_{10}-\left(\frac{23}{6}\right) x_{11} \\
& S_{21}=\left(\frac{98}{57}\right)-\left(\frac{36}{19}\right) x_{1}+\left(\frac{20}{19}\right) x_{2}-\left(\frac{103}{19}\right) x_{4}-\left(\frac{166}{57}\right) x_{6}+\left(\frac{118}{57}\right) x_{8}-\left(\frac{157}{57}\right) x_{9}-\left(\frac{188}{57}\right) x_{10}+\left(\frac{1}{3}\right) x_{11} \\
& -\left(\frac{31}{57}\right) S_{13}+\left(\frac{1}{114}\right) S_{15}-\left(\frac{5}{114}\right) S_{18}+\left(\frac{35}{38}\right) S_{20} \\
& +\left(\frac{46}{57}\right) S_{18}+\left(\frac{16}{57}\right) S_{20}
\end{aligned}
$$

For $i=0$, equation (13) becomes

$245 x_{1}+170 x_{2}+141 x_{4}+429 x_{6}+102 x_{8}+312 x_{9}+468 x_{10}+114 x_{11}+28 S_{13}+40 S_{15}+85 S_{18}+S_{20}=20$

The only solution is when all variables are zero and $S_{20}=20$.

However, this solution does not lead to an integer solution as basic variables do not satisfy integer requirements.

When $i=1$, the RHS of the CE (13) becomes 77, and the equation (13) for integer solution purposes reduces to $28 s_{13}+40 s_{15}+s_{20}=77$. There are four possible solutions.

(i) $s_{20}=77$, leads to infeasible solution.

(ii) $s_{15}=1, s_{20}=37$, leads to infeasible solution. 
International Journal of Mathematical, Engineering and Management Sciences

Vol. 2, No. 1, 1-16, 2017

https://dx.doi.org/10.33889/IJMEMS.2017.2.1-001

(iii) $s_{13}=s_{15}=1, \quad s_{20}=9$, leads to $x_{3}=3, x_{5}=5, x_{7}=2$, which is feasible and $\mathrm{Z}_{\mathrm{PIP}}=43$.

(iv) $s_{13}=2, s_{20}=21$, leads to infeasible solution.

Thus, we have $44.351 \leq Z_{M I P} \leq 43$. We substitute integer values of the integer restricted variables $x_{1}, x_{3}$ and $x_{6}$, and the modified LP becomes

$\operatorname{Max} 15-x_{2}+3 x_{4}+4 x_{5}+2 x_{7}+3 x_{8}+2 x_{9}-3 x_{10}+x_{11}+2 x_{12}$

subject to :

$4 x_{2}-1 x_{4}+5 x_{5}-2 x_{7}+4 x_{8}+0 x_{9}+0 x_{10}+3 x_{11}+1 x_{12} \leq 24$

$3 x_{5}+3 x_{7}+x_{10}+4 x_{11} \leq 26$

$4 x_{8}+x_{10}+2 x_{12} \leq 5$

$1 x_{2}+3 x_{4}+2 x_{5}+3 x_{7}-2 x_{8}+4 x_{9}+2 x_{11} \leq 30$

$2 x_{2}-2 x_{5}+x_{10}-2 x_{11} \leq 10$

$4 x_{4}+x_{5}+2 x_{7}++5 x_{9}+3 x_{10}+x_{11} \leq 9$

$2 x_{2}+x_{4}+x_{7}+2 x_{8}+4 x_{11}+5 x_{12} \leq 28$

$x_{2}+3 x_{5}+x_{8}+1 x_{9}+2 x_{10}+2 x_{11}+6 x_{12} \leq 36$

$2 x_{4}+x_{5}-2 x_{7}-x_{9}+2 x_{10}+3 x_{12} \leq 12$

$x_{2}, x_{4}, x_{5}, x_{7}, x_{8}, x_{9}, x_{10}, x_{11}, x_{12} \geq 0$

Solving (23) as an LP model gives

$x_{2}=0, x_{4}=0, x_{5}=\frac{61}{12}, x_{7}=\frac{47}{24}, x_{8}=0, x_{9}=0, x_{10}=0, x_{11}=0, x_{12}=2.5$

Combining the integer solution and the continuous solutions we have the optimal MIP solution as $x_{1}=0, x_{2}=0, x_{3}=3, x_{4}=0, x_{5}=\frac{61}{12}, x_{6}=0, x_{7}=\frac{47}{24}, x_{8}=0, x_{9}=0, x_{10}=0, x_{11}=0, x_{12}=2.5$ and $Z=44.25$.

Thus, we have a feasible solution to the given MIP and its current bounds are: UB $=44.351, \mathrm{LB}=$ 43. Since the difference between the current value and the UB is insignificant, the former approximately constitutes the required optimal solution.

\section{Conclusion}

\subsection{Advantages}

The proposed method will have several advantages over existing techniques and some of them include, but are not limited to

- There is no round off errors since rounding leads to non-optimal or non-feasible solutions.

- The dimension of the matrix $A$, constituting the coefficients of the decision variables in the constraints, remains unchanged. 
International Journal of Mathematical, Engineering and Management Sciences

Vol. 2, No. 1, 1-16, 2017

https://dx.doi.org/10.33889/IJMEMS.2017.2.1-001

- There are no sub-problems as is in the case of branch and bound or branch and cut methods.

- The method does not depend on the initial relaxed solution, but uses the relaxed solution as the upper bound.

- The method searches the optimal solution using the simplex algorithm, but moves over the integer polyhedron.

- This approach is suitable for changes in the input values.

- It utilises and makes use of the characteristic equation to adjust the lower bound.

\subsection{Further Challenges}

A solution of a CE can be demanding for larger values of $i$. Further work is required on how to solve a CE. The method uses the concept of ordered solutions, but for most of the ordered solutions, the value of $i$ will be high. For high values of $i$, the solution to the CE becomes more demanding.

In the branch and bound approach for a MIP, when a large number of variables are integer restricted, the number of sub-problems can be very high. Sometimes even a feasible solution is not easily obtainable. However, the proposed approach guarantees determination of a feasible solution and also gives its bounds. The proposed approach works better when integer restricted variables are relatively large (see Example 1 above).

The branch and bound technique may work better if integer restricted variables are only a few in number. In fields like machine learning, computer vision, advertising, and statistics, it is quite common to encounter MIP formulations with millions of binary decision variables. These algorithms can yield good results in practice but do not offer any theoretical bounds on runtime and solution quality. The proposed method may prove to be useful for these problems.

\section{References}

Benders, J. F. (1962). Partitioning procedures for solving mixed-variables programming problems. Numerische Mathematik, 4(1), 238-252.

Borsani, V., Matta, A., Beschi, G., \& Sommaruga, F. (2006, October). A home care scheduling model for human resources. In 2006 International Conference on Service Systems and Service Management (Vol. 1, pp. 449-454). IEEE.

Bredstrom, D., \& Rönnqvist, M. (2007). A branch and price algorithm for the combined vehicle routing and scheduling problem with synchronization constraints. NHH Dept. of Finance \& Management Science Discussion Paper, (2007/7).

Burer, S., \& Letchford, A. N. (2012). Non-convex mixed-integer nonlinear programming, a survey. Surveys in Operations Research and Management Science, 17(2), 97-106.

Dey, S. S., \& Vielma, J. P. (2010, June). The Chvátal-Gomory closure of an ellipsoid is a polyhedron. In International Conference on Integer Programming and Combinatorial Optimization (pp. 327-340). Springer Berlin Heidelberg.

Dohn, A., Kolind, E., \& Clausen, J. (2009). The manpower allocation problem with time windows and jobteaming constraints: A branch-and-price approach. Computers \& Operations Research, 36(4), 11451157.

Fiorini, S., Massar, S., Pokutta, S., Tiwary, H. R., \& de Wolf, R. (2012, May). Linear vs. semidefinite extended formulations, exponential separation and strong lower bounds. In Proceedings of the fortyfourth annual ACM symposium on Theory of Computing (pp. 95-106). ACM. 
International Journal of Mathematical, Engineering and Management Sciences

Vol. 2, No. 1, 1-16, 2017

https://dx.doi.org/10.33889/IJMEMS.2017.2.1-001

Geoffrion, A. M., \& Marsten, R. E. (1972). Integer programming algorithms, A framework and state-of-theart survey. Management Science, 18(9), 465-491.

Hillier, F.S. and Lieberman, G.J. (2001), Introduction to Operations Research, McGraw Hill, New York.

Jozefowiez, N., Laporte, G., \& Semet, F. (2012). A generic branch-and-cut algorithm for multiobjective optimization problems: Application to the multilevel traveling salesman problem. INFORMS Journal on Computing, 24(4), 554-564.

Kaibel, V. (2011). Extended formulations in combinatorial optimization. arXiv preprint arXiv:1104.1023.

Kumar, S. and Munapo, E. (2012). Some Lateral Ideas and their applications for developing new solution procedures for a pure integer programming model, Keynote address, Proc. Of the Herbal International Conference on Applications of Mathematics and Statistics - Intelligent solutions through Mathematics and Statistics, Edited by Mariappan, Srinivasan and Amritraj, Excel India Publisher, pp 13-21, January.

Kumar, S., Munapo, E. and Jones, B.C. (2007). An integer equation controlled descending path to a protean pure integer program. Indian Journal of Mathematics, 49(2), 211-237.

Laesanklang, W., Landa-Silva, D. and Castillo-Salazar, J.A. (2015). Mixed integer programming with decomposition to solve a workforce scheduling and routing problem. International Conference on Operations Research and Enterprise Systems.

Morán, R., Diego, A., Santanu, S. Dey and Vielma, J. P. (2012). A strong dual for conic mixed-integer programs. SIAM Journal on Optimization, 22(3), 1136-1150.

Noraini, M.R. and Geraghty, J. (2011). Genetic Algorithm Performance with Different Selection Strategies in Solving TSP, Proceedings of the World Congress on Engineering, July 6-8, London, UK.

Rasmussen, M. S., Justesen, T., Dohn, A., \& Larsen, J. (2012). The home care crew scheduling problem: Preference-based visit clustering and temporal dependencies. European Journal of Operational Research, 219(3), 598-610.

Sen, S., \& Sherali, H. D. (2006). Decomposition with branch-and-cut approaches for two-stage stochastic mixed-integer programming. Mathematical Programming, 106(2), 203-223.

Sherali, H. D. and Adams, W. P. (1990). A hierarchy of relaxations between the continuous and convex hull representations for zero-one Programming problems. SIAM Journal on Discrete Mathematics, 3(3), 411430 .

Sourd, F. and Spanjaard, O. (2008). A multiobjective branch-and-bound framework: Application to the biobjective spanning tree problem. INFORMS Journal on Computing, 20(3),472-484.

Sridhar, S. (2014). Mixed-Integer programming approaches for some non-convex and combinatorial optimization problems (Doctoral dissertation, Stanford University).

Vielma, J. P. (2015). Mixed integer linear programming formulation techniques. Society for Industrial and Applied Mathematics, 57(1), 3-57.

Yıldiz, S., \& Vielma, J. P. (2013). Incremental and encoding formulations for mixed integer programming. Operations Research Letters, 41(6), 654-658. 
International Journal of Mathematical, Engineering and Management Sciences

Vol. 2, No. 1, 1-16, 2017

https://dx.doi.org/10.33889/IJMEMS.2017.2.1-001

\section{Appendices}

\begin{tabular}{|c|c|c|c|}
\hline Variable & Value & Objective Cost & Reduced Cost \\
\hline$x_{1}$ & 0 & 2 & $245 / 57$ \\
\hline$x_{2}$ & 0 & -1 & $170 / 57$ \\
\hline$x_{3}$ & $148 / 57$ & 5 & 0 \\
\hline$x_{4}$ & 0 & 3 & $47 / 19$ \\
\hline$x_{5}$ & $248 / 57$ & 4 & 0 \\
\hline$x_{6}$ & 0 & -2 & $143 / 19$ \\
\hline$x_{7}$ & $42 / 19$ & 2 & 0 \\
\hline$x_{8}$ & 0 & 3 & $34 / 19$ \\
\hline$x_{9}$ & 0 & 2 & $104 / 19$ \\
\hline$x_{10}$ & 0 & -3 & $156 / 19$ \\
\hline$x_{11}$ & 0 & 1 & 2 \\
\hline$x_{12}$ & $200 / 57$ & 3 & 0 \\
\hline
\end{tabular}

Table 3. Solution of LP of example 2

Using LIPS programme, the LP solution found is shown in Table 4. 
International Journal of Mathematical, Engineering and Management Sciences Vol. 2, No. 1, 1-16, 2017

https://dx.doi.org/10.33889/IJMEMS.2017.2.1-001

\begin{tabular}{|c|c|c|c|c|c|c|c|c|c|c|c|c|c|c|c|c|c|c|c|c|c|c|}
\hline Basis & $\times 1$ & $\times 2$ & $\times 3$ & $\times 4$ & $\times 5$ & $\times 6$ & $\times 7$ & $\times 8$ & $\times 9$ & $\times 10$ & $\times 11$ & $\times 12$ & s13 & s14 & s15 & s16 & s17 & s18 & s19 & s20 & S21 & RHS \\
\hline$\times 5$ & $7 / 19$ & $13 / 19$ & 0 & $10 / 19$ & 1 & $-11 / 57$ & 0 & $31 / 57$ & $49 / 57$ & $26 / 57$ & $2 / 3$ & 0 & $10 / 57$ & 0 & $-2 / 57$ & 0 & 0 & $10 / 57$ & 0 & $-1 / 57$ & 0 & $284 / 57$ \\
\hline S14 & $-223 / 114$ & $-121 / 114$ & 0 & $-130 / 19$ & 0 & $-41 / 19$ & 0 & $-47 / 38$ & $-168 / 19$ & $-81 / 19$ & 1.5 & 0 & $-16 / 57$ & 1 & $-5 / 114$ & 0 & 0 & $-203 / 114$ & 0 & $7 / 114$ & 0 & $298 / 57$ \\
\hline$\times 3$ & $47 / 57$ & $4 / 57$ & 1 & $2 / 19$ & 0 & $17 / 19$ & 0 & $16 / 19$ & $2 / 19$ & $3 / 19$ & 0 & 0 & $2 / 57$ & 0 & $11 / 57$ & 0 & 0 & $2 / 57$ & 0 & $-4 / 57$ & 0 & $148 / 57$ \\
\hline S16 & $39 / 38$ & $29 / 38$ & 0 & $-59 / 19$ & 0 & $-32 / 57$ & 0 & $-115 / 114$ & $-215 / 57$ & $-256 / 57$ & $1 / 6$ & 0 & $-2 / 57$ & 0 & $35 / 114$ & 1 & 0 & $-175 / 114$ & 0 & $-11 / 114$ & 0 & $164 / 57$ \\
\hline S17 & $-142 / 57$ & $176 / 57$ & 0 & $12 / 19$ & 0 & $-55 / 57$ & 0 & $-130 / 57$ & $74 / 57$ & $73 / 57$ & $-2 / 3$ & 0 & $4 / 19$ & 0 & $-16 / 19$ & 0 & 1 & $4 / 19$ & 0 & $14 / 57$ & 0 & $410 / 19$ \\
\hline$x 7$ & $47 / 114$ & $-43 / 114$ & 0 & $32 / 19$ & 0 & $37 / 57$ & 1 & $-79 / 114$ & $115 / 57$ & $68 / 57$ & $1 / 6$ & 0 & $-2 / 19$ & 0 & $-3 / 38$ & 0 & 0 & $15 / 38$ & 0 & $5 / 114$ & 0 & $42 / 19$ \\
\hline S19 & $-37 / 114$ & $371 / 114$ & 0 & $12 / 19$ & 0 & $2 / 57$ & 0 & $367 / 114$ & $-40 / 57$ & $-98 / 57$ & $23 / 6$ & 0 & $31 / 57$ & 0 & $-1 / 114$ & 0 & 0 & $5 / 114$ & 1 & $-35 / 38$ & 0 & $470 / 57$ \\
\hline$\times 12$ & $-1 / 57$ & $-10 / 57$ & 0 & $\begin{array}{l}-5 / 19 \\
\end{array}$ & 0 & $5 / 19$ & 0 & $-2 / 19$ & $-5 / 19$ & $2 / 19$ & 0 & 0 & $-5 / 57$ & 0 & $1 / 57$ & 0 & 0 & $-5 / 57$ & 0 & $10 / 57$ & 0 & $200 / 57$ \\
\hline S21 & $36 / 19$ & $\begin{array}{l}-1 / 1 / 19 \\
-20 / 19\end{array}$ & 0 & $\begin{array}{l}103 / 19 \\
\end{array}$ & 0 & $166 / 57$ & 0 & $\frac{-2 / 17}{-118 / 57}$ & $\frac{-3 / 1}{157 / 57}$ & $188 / 57$ & $-1 / 3$ & 0 & $\begin{array}{l}-11 / 57 \\
\end{array}$ & 0 & $\frac{1 / J 1}{-32 / 57}$ & 0 & 0 & $46 / 57$ & 0 & $-16 / 57$ & 1 & $98 / 57$ \\
\hline Obj. & $-245 / 57$ & $-170 / 57$ & 0 & $-47 / 19$ & 0 & $-143 / 19$ & 0 & $-34 / 19$ & $-104 / 19$ & $-156 / 19$ & -2 & 0 & $-28 / 57$ & 0 & $-40 / 57$ & 0 & 0 & $-85 / 57$ & 0 & $-5 / 57$ & 0 & $2528 / 57$ \\
\hline
\end{tabular}

Table 4. Final table of the Simplex iterations

$Z=44.35087719$. 